\title{
IMAP.2
}

\section{An automatic imaging spectroscopic ellipsometer for characterization of nano-film pattern on solid substrate}

\author{
Y.H. Meng ${ }^{1,2}$, Y.Y. Chen ${ }^{1}$, C. $\mathrm{Qi}^{1,2}$, L. Lui ${ }^{1,2}$, and G. Jin ${ }^{1}$ \\ ${ }^{1}$ Institute of Mechanics, Chinese Academy of Sciences, Beijing, P.R.China \\ ${ }^{2}$ Graduate School of Chinese Academy of Sciences, Beijing, P.R.China
}

An auto imaging spectroscopic ellipsometer (ISE) is presented, which is able to characterize the spatial distribution information of the nano-film pattern, such as the physical, structural and spatial properties including dielectric function, film thickness distribution, surface roughness, etc. The ISE integrates advantages of the imaging ellipsometer and the spectroscopic ellipsometer, which may provide the spectroscopic ellipsometric parameters $\Psi(\lambda)$ and $\Delta(\lambda)$ images of the film pattern with $\mu \mathrm{m}$ resolution in continuous spectrum of $400-650 \mathrm{~nm}$.

Figure 1 gives the schematic diagram of the ISE, which based on polarizer - compensator specimen - analyzer (PCSA) configuration. A Xenon short arc lamp emits stable outgoing light flux with continuous spectrum. After converged by an achromatic lens, the white light is dispersed into single wavelength light beam by a monochromator. A collimating lens system after monochromator is used to provide expanded parallel beam in a diameter of $15 \mathrm{~mm}$ as a probe. In order to make the intensity distribution of light source and optics in spectrum fit the dynamic response range of the sensor, an auxiliary polarizer $\mathrm{Px}$ is set before the polarizer with changing its azimuth angle in respect to the polarizer. The auxiliary polarizer, polarizer, compensator and analyzer are positioned in hollow precision rotators driven by motors for azimuth modulation in respect to the incidence plane. Specimen is held on a stage by negative pressure and aligned by an optical self-collimated telescope. An achromatic lens images the specimen onto a video CCD camera, of which the electronic gain is calibrated. The signal is transferred to an $\mathrm{A} / \mathrm{D}$ converter in computer and the ellipsometric images are recorded in grayscale format. In order to adjust the lateral resolution and the field of view on specimen, the optical magnification could be modulated. For imaging ellipsometry, the image plane is not perpendicular to the optical axis, so an auto-focusing method is developed, which combines focusing in optical axes direction and angle-tilted focusing by step moving and rotating imaging sensor successively. The rotating-compensator sampling method is used for the quantitative measurement due to its advantages: (a) non-ambiguous determination of ellipsometric angle $\Delta$, (b) insensitivity to source and detector polarization, (c) no need for a DC level, and (d) self-calibrating. In the performance of measurement, the azimuth of compensator is auto-controlled step by step. At each sampling position, the CCD camera successively grabs many images and averages these to reduce the random noise. The ellipsometric parameters $\Psi(\lambda)$ and $\Delta(\lambda)$ in each pixel of images with wavelength are deduced with Fourier analysis method. The systematic errors is effectively reduced by twozone method, which means to average the results achieved under the azimuth angle of analyzer at $A=45^{\circ}$ and $A=-45^{\circ}$, respectively. As a demonstration of the specifications of

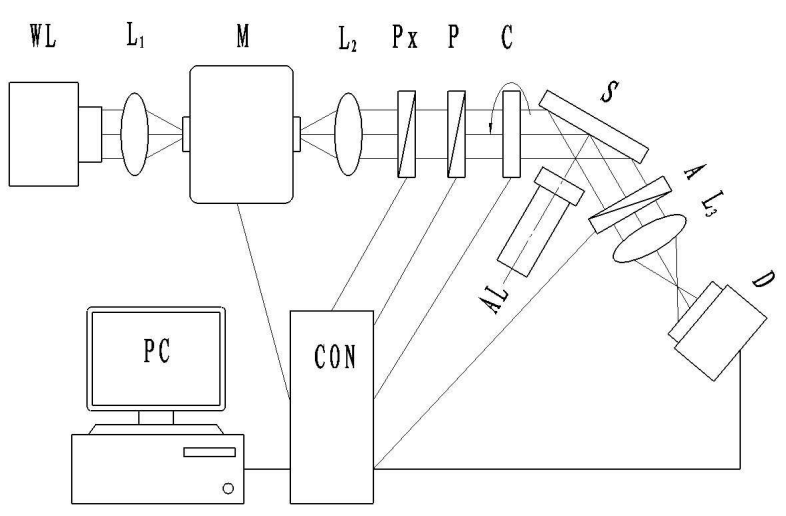

Figure 1: Schematic diagram of imaging spectroscopic ellipsometer WL-White lamp, $\mathrm{L}_{1}, \mathrm{~L}_{2}, \mathrm{~L}_{3}$-Lens, MMonochromator, Px-Auxiliary polarizer, P-Polarizer, C-Compensator, S-Specimen, AL-Alignment telescope, A-Analyzer, D-CCD camera, CON-Controller, PCComputer. ISE, a $\mathrm{SiO}_{2}$ film pattern on $\mathrm{Si}$ substrate is measured for the spectroscopic ellipsometric parameters $\Psi(\lambda)$ and $\Delta(\lambda)$ images with $\mu$ m lateral resolution, with which the spatial and structural properties of each micro spot were further deduced in $0.1 \mathrm{~nm}$ thickness resolution. 